\title{
EXCITONS IN NANOSCALE SYSTEMS
}

GREGORY D. SCHOLES AND GARRY RUMBLES

Nature Materials 5, 683-696 (2006).

In this Review Article, the spelling of the first author's name in ref. 74, and in the citation of this reference within the text on page 691 , was incorrect. It should have read Zhao. 